\title{
Receding Horizon optimal control for the wave equation
}

\author{
Nils Altmüller, Lars Grüne, Karl Worthmann
}

\begin{abstract}
We present a receding horizon optimal control approach for the one-dimensional linear wave equation. In order to prove stability and suboptimality of this approach we use a weighted energy as cost functional. Furthermore we apply similar methods to a semilinear parabolic equation.
\end{abstract}

\section{INTRODUCTION}

Receding horizon control (RHC), also known as model predictive control (MPC), is a well established technique in order to deal with optimal control problems on an infinite time horizon. The main idea of this method consists of approximating the solution on the infinite time horizon by iteratively solving problems on a finite time horizon. To this end, an optimal control on the finite time horizon is computed for the current state of the system whose first part is applied to the system. This procedure is then repeated iteratively by shifting the optimization horizon forward for the elapsed time and repeating the optimization with the new state. This procedure generates a static state feedback for the original problem and is applicable - among others - for systems governed by ordinary [11] and partial differential equations [8].

In the latter case still a lot of problems are unsolved even for the linear case. Since the computational complexity of RHC schemes grows rapidly with the length of the optimization horizon, estimates for minimal stabilizing horizons are of particular interest. Here, we focus on a linear hyperbolic and a semilinear parabolic equation and deduce such bounds explicity.

First, we investigate stability properties of a receding horizon controller for the one dimensional wave equation. It is known that MPC stabilizes this problem if suitable terminal constraints are added, cf. [8]. However, numerical results suggest that such constraints are actually not needed in order to enforce stability, cf. [7]. Motivated by these results, in this paper we investigate the MPC scheme without stabilizing terminal constraints and prove stability for suitably designed running costs - even for very short optimization horizons. To this end, we establish a controllability condition from [5] and exploit a formula from [6] which is based on this condition. This allows for giving explicit bounds for the minimal stabilizing horizon.

As an extension we consider a semilinear parabolic PDE. In [2] it has been shown how the suboptimality theory from [5] can be applied in order to derive design guidelines for the running costs which allow for reducing the optimization horizon in the control strategy. In contrast to the heuristic arguments in this reference, here we deduce rigorous estimates on the required horizon length.
The paper is organized as follows: in Section II we describe the setup and briefly summarize needed results from [2]. In the ensuing sections we give stability and suboptimality results for the boundary controlled wave equation as well as for a semilinear parabolic equation (heat equation). In Section V we illustrate our results numerically.

\section{SETUP AND PRELIMINARIES}

We consider a nonlinear discrete time control system given by

$$
x(n+1)=f(x(n), u(n)), \quad x(0)=x_{0}
$$

with $x(n) \in X$ and $u(n) \in U . X$ and $U$ are arbitrary metric spaces with metric $d(\cdot, \cdot)$. With regard to PDEs the state space $X$ and the control value space $U$ are appropriate function spaces with suitable norms. Here we denote the space of control sequences $u: \mathbb{N}_{0} \rightarrow U$ by $\mathcal{U}$ and the solution trajectory for some $u \in \mathcal{U}$ by $x_{u}(n)$.

An important class of discrete time systems are sampleddata systems induced by a controlled (partial) differential equation with sampling period $T>0$ where the discrete time control $u(n)$ corresponds to the continuous time control $u(\cdot)$ in the sampling interval $[n T,(n+1) T)$.

Our goal is to minimize the infinite time horizon cost functional

$$
J_{\infty}\left(x_{0}, u\right)=\sum_{n=0}^{\infty} l\left(x_{u}(n), u(n)\right)
$$

with running cost $l: X \times U \rightarrow \mathbb{R}_{0}^{+}$in order to obtain a feedback control law $\mu: X \rightarrow U$. The optimal value function is given by

$$
V_{\infty}\left(x_{0}\right):=\inf _{u \in \mathcal{U}} J_{\infty}\left(x_{0}, u\right)
$$

Solving this infinite time horizon problem is in general computationally infeasible. Thus we use a receding horizon approach in order to compute an approximately optimal feedback law: at time $n$ for the current state $x(n)$ we solve the finite time horizon optimal control problem

$$
\underset{u(\cdot)}{\operatorname{minimize}} J_{N}(x(n), u)=\sum_{k=0}^{N-1} l(x(n+k), u(n+k))
$$

in order to obtain the optimal contol sequence $u^{*}(n), u^{*}(n+$ $1), \ldots, u^{*}(n+N-1)$. We implement only the first element of this control sequence in order to get a feedback $\mu_{N}(x(n)):=$ $u^{*}(n)$ and the next state $x(n+1)$. Iterative application of this procedure provides a control sequence on the infinite time horizon. $N \in \mathbb{N}_{\geq 2}$ denotes the optimization horizon and the optimal value function for the finite time horizon is given by

$$
V_{N}\left(x_{0}\right):=\inf _{u \in \mathcal{U}} J_{N}\left(x_{0}, u\right)
$$


We obtain a closed loop representation by applying the map $\mu_{N}: X \rightarrow U$ to (1)

$$
x_{\mu}(n+1)=f\left(x_{\mu}(n), \mu\left(x_{\mu}(n)\right)\right) .
$$

In many papers in the receding horizon literatur additional stabilizing terminal constraints or terminal costs are added to the optimization objective (2) to ensure stability of the closed loop system, cf. [8]. In contrast to that we consider (2) without any changes. This is motivated by the fact that this version of the receding horizon approach is easier to implement and appears to be predominant in practical applications, cf. [3], and that numerical simulations suggest that it is able to stabilize the wave equation, cf. [7]. Moreover, for this unconstrained approach suboptimality estimates can be proven, cf. [5].

We briefly summarize some stability and suboptimality results which can be found in [6].

Proposition 1: Assume that there exists $\alpha \in(0,1]$ such that for all $x \in X$ the relaxed Lyapunov inequality

$$
V_{N}(x) \geq V_{N}\left(f\left(x, \mu_{N}(x)\right)\right)+\alpha l\left(x, \mu_{N}(x)\right)
$$

holds. Then for all $x \in X$ the estimate

$$
\alpha V_{\infty}(x) \leq \alpha J_{\infty}\left(x, \mu_{N}\right) \leq V_{N}(x) \leq V_{\infty}(x)
$$

holds. If in addition, there exist $x^{*} \in X$ and $\mathcal{K}_{\infty}$-functions ${ }^{1}$ $\alpha_{1}, \alpha_{2}$ such that the inequalities

$$
\begin{aligned}
l^{*}(x):=\min _{u \in U} l(x, u) & \geq \alpha_{1}\left(d\left(x, x^{*}\right)\right) \text { and } \\
\alpha_{2}\left(d\left(x, x^{*}\right)\right) & \geq V_{N}(x)
\end{aligned}
$$

hold for all $x \in X$, then $x^{*}$ is a globally asymptotically stable equilibrium for (3) with feedback $\mu=\mu_{N}$ and Lyapunov function $V_{N}$.

In order to estimate $\alpha$ in (4) we require the following controllability property:

Definition 1: We call the system (1) exponentially controllable with respect to the running cost $l$ if there exist constants $C \geq 0$ (overshoot bound) and $\sigma \in[0,1)$ (decay rate) such that for each $x \in X$ there exists $u_{x} \in \mathcal{U}$ with

$$
l\left(x_{u_{x}}(n), u_{x}(n)\right) \leq C \sigma^{n} l^{*}(x)
$$

Remark 1: Note that exponential controllability with respect to the running costs is not as restrictive as it seems. Since the running costs can be used as a design parameter, this includes even systems which are only asymptotically controllable, cf. [6].

Theorem 1: Assume that the sytem (1) and $l$ satisfy the controllability condition (8) and let the optimization horizon $N$ be given. Then the suboptimality degree $\alpha_{N}$ from (4) is given by

$$
\alpha_{N}:=\alpha=1-\frac{\left(\gamma_{N}-1\right) \prod_{i=2}^{N}\left(\gamma_{i}-1\right)}{\prod_{i=2}^{N} \gamma_{i}-\prod_{i=2}^{N}\left(\gamma_{i}-1\right)}
$$

with $\gamma_{i}=C\left(1-\sigma^{i}\right) /(1-\sigma)$.

\footnotetext{
${ }^{1}$ A function $\alpha: \mathbb{R}^{+} \rightarrow \mathbb{R}^{+}$is said to be of class $\mathcal{K}_{\infty}$ if it is continuous, strictly increasing, and unbounded with $\alpha(0)=0$
}

As a consequence of Theorem 1 the closed loop system (3) is asymptotically stable and the suboptimality estimate (5) holds whenever $\alpha$ is positive and Equations (6)-(7) are satisfied. For negative $\alpha$ there exist a system (1) and running costs $l$ which satisfy (6)-(8) but for which (3) with $\mu=\mu_{N}$ is not asymptotically stable, cf. [5].

The minimal horizon that guarantees stability is the smallest $N \in \mathbb{N}_{N \geq 2}$ with $\alpha_{N}>0$.

\section{RHC OF THE WAVE EQUATION}

In the following we will change the notation to be consistent with the usual PDE notation: $x \in \Omega$ is the independent space variable while the unknown function $y(t, \cdot): \Omega \rightarrow \mathbb{R}$ is the state now.

\section{A. Problem formulation}

We consider the one-dimensional linear wave equation with homogeneous Dirichlet boundary condition on the left and Neumann boundary control on the right boundary

$$
\begin{aligned}
y_{t t}(x, t)-c^{2} y_{x x}(x, t) & =0 & & \text { on }(0, L) \times(0, \infty) \\
y(0, t) & =0 & & \text { on }(0, \infty) \\
y_{x}(L, t) & =u(t) & & \text { on }(0, \infty)
\end{aligned}
$$

Here $c \neq 0$ denotes the propagation speed of the wave. The initial data are given by $y(x, 0)=y_{0}(x)$ and $y_{t}(x, 0)=$ $y_{1}(x)$ with $\left(y_{0}, y_{1}\right) \in C([0, L]) \times L^{2}([0, L])$. The solution space is given by

$$
\begin{aligned}
X=\{y: y & \in L^{2}\left(0, t^{*} ; H^{1}([0, L])\right) \text { with } \\
y_{t} & \left.\in L^{2}\left(0, t^{*} ; L^{2}([0, L])\right), \forall t^{*}>0\right\}
\end{aligned}
$$

and $u \in L^{\infty}([0, \infty])$. We aim at steering the system to the origin $y \equiv 0$ which is an equilibrium for (10)-(12). It is well known that this evolution equation is exactly controllable in finite time for $\bar{T} \geq 2 L / c$, cf. [13]. However, we are interested in the receding horizon approach with sampling time $T>0$ and optimization horizon $(N-1) T, N \in \mathbb{N}_{\geq 2}$ with $(N-1) T \ll \bar{T}$. The numerical results in [7] show that this approach works well even for $N=2$ which is in our setting the shortest possible horizon. This special case is in the literatur also termed instantaneous control.

Our cost functional is given by

$$
\sum_{n=0}^{N-1} \frac{1}{4} \int_{0}^{L} \rho\left(y_{x}(x, n T), y_{t}(x, n T)\right) d x+\lambda \int_{0}^{N T} u(t) d t
$$

with

$$
\begin{aligned}
\rho\left(y_{x}(\cdot, t), y_{t}(\cdot, t)\right) & =\omega_{1}(\cdot)\left(y_{x}(\cdot, t)+y_{t}(\cdot, t) / c\right)^{2} \\
& +\omega_{2}(\cdot)\left(y_{x}(\cdot, t)-y_{t}(\cdot, t) / c\right)^{2}
\end{aligned}
$$

Here $\omega_{i}:[0, L] \rightarrow \mathbb{R}, i=1,2$ denote appropriate weight functions.

Remark 2: Note that our cost functional consists of two parts. The first is related to the system's energy and evaluates the motion to the left and the right boundary separately, e.g. $\left(y_{x}(\cdot, t)+y_{t}(\cdot, t) / c\right)$ represents the movement to the left boundary. For the special case $\omega_{1}=\omega_{2} \equiv 1$ we obtain 
exactly the energy of the system. However - as it will be shown in Section V - the classical energy is not useful in order to show instantaneous controllability.

For our purpose we choose the weight functions

$$
\omega_{1}(x):=1+L+x \quad \text { and } \quad \omega_{2}(x):=1+L-x .
$$

The second term in (13) penalizes the control effort with weight $\lambda>0$.

\section{B. Stability estimates}

Since Proposition 1 and Theorem 1 are formulated in a discrete time setting we rewrite the continuous-time system as

$$
y(n+1)=f(y(n), u(n))
$$

with state $y(n):=y(\cdot, n T)$ and control $u(n) \in U:=$ $L^{\infty}([0, T), \mathbb{R})$. This enables us to apply the results from Section II in order to prove stability of the receding horizon closed loop, i.e., that the MPC feedback steers the system to its equilibrium. Here the discrete time $n$ corresponds to the continuous time $n T$. Hence the running costs $l(y(n), u(n))$ are given by

$$
\frac{1}{4} \int_{0}^{L} \rho\left(y_{x}(x, n T), y_{t}(x, n T)\right)+\lambda \int_{0}^{T} u(n)(t)^{2} d t
$$

with $\omega_{1}$ and $\omega_{2}$ from (14).

In order to estimate the parameters $C$ and $\sigma$ from our controllability condition given in Definition 1 we choose the particular control

$$
u(n):=\frac{1}{2}\left(y_{x}(L-c t, n T)-\frac{y_{t}(L-c t, n T)}{c}\right)
$$

which ensures that there do not occur any reflections on the right boundary. By using this control the solution of (10)-(12) coincides with the uncontrolled solution of the wave equation on a semi-infinite interval $[0, \infty)$, cf. [1]. The corresponding solution can be calculated by D'Alembert's method, cf. [12],

$$
\begin{aligned}
y(x, t) & =\frac{1}{2}\left(y_{0}(x+c t)+y_{0}(x-c t)\right) \\
& +\frac{1}{2 c} \int_{x-c t}^{x+c t} y_{1}(s) d s \text { for } x>c t, \\
y(x, t) & =\frac{1}{2}\left(y_{0}(c t+x)-y_{0}(c t-x)\right) \\
& +\frac{1}{2 c} \int_{c t-x}^{c t+x} y_{1}(s) d s \text { for } x<c t .
\end{aligned}
$$

Note that we do not require optimality of the control (16) in our approach. We begin with estimating the overshoot constant $C$ from Inequality (8) for the running costs defined in (15) and the control which is specified in (16):

$$
\begin{aligned}
& l(y(n), u(n)) \\
& =\frac{1}{4} \int_{0}^{L} \omega_{1}(x)\left(y_{x}(x, n T)+y_{t}(x, n T) / c\right)^{2} \\
& \quad+\omega_{2}(x)\left(y_{x}(x, n T)-y_{t}(x, n T) / c\right)^{2} d x
\end{aligned}
$$

$$
\begin{aligned}
& +\frac{\lambda}{4} \int_{0}^{T}\left(y_{x}(L-c t, n T)-y_{t}(L-c t, n T) / c\right)^{2} d t \\
= & l^{*}(y(n T))+\frac{\lambda}{4 c} \int_{L-c T}^{L}\left(y_{x}(x, n T)-y_{t}(x, n T) / c\right)^{2} d t \\
\leq & (1+\lambda / c) l^{*}(y(n T))=C l^{*}(y(n T))
\end{aligned}
$$

with $C:=(1+\lambda / c)$. Here we have used the property $\omega_{i} \geq 1$, $i=1,2$, of our weight functions from (14). Next we show the inequality

$$
l^{*}(y(i+1)) \leq \sigma l^{*}(y(i))
$$

with decay rate $\sigma \in[0,1)$. This inequality is equivalent to $(1-\eta) l^{*}(y(i)) \geq l^{*}(y(i+1))$ with $\eta:=1-\sigma$. Hence, it is sufficient to establish the inequality

$$
l^{*}(y(i))-l^{*}(y(i+1)) \geq \eta l^{*}(y(i)) .
$$

in order to show the desired inequality. The validity of Inequality (20) with $\eta=c T /(1+2 L)$ has been proven in [1]. The decisive tools in order to establish this relation are the explicit formulas given from (17) for the control defined in (16). As a consequence, we obtain $\sigma=1-c T /(1+2 L)$. This implies - in combination with our estimate for the overshoot $C$ derived in Inequality (18) - exponential controllability in terms of the running costs, i.e.,

$$
l(y(n), u(n)) \leq C l^{*}(y(n)) \leq C \sigma^{n} l^{*}(y(0)) .
$$

Hence, we have shown the validity of the controllability condition given in Definition 1.

Remark 3: The decrease implied by $\sigma$ depends only on the chosen weight functions. In addition, there occurs an energy loss in the amount of

$$
\int_{L-c T}^{L} \omega_{2}(x+c T)\left[y_{0}^{\prime}(x)-y_{1}(x) / c\right]^{2} d x
$$

This represents the energy which is removed by means of the boundary control.

Since we have deduced explicit expressions for the overshoot $C$ and the decay rate $\sigma$ in (8), we are able to utilize Theorem 1 in order to show stability of the receding horizon feedback for optimization horizon $N=2$, i.e., instantaneous control. For stability we need $\alpha_{2}>0$ with $\alpha_{2}=1-(C(1+$ $\sigma)-1)^{2}$ given by Formula (9). Thus, Theorem 1 guarantees stability for

$$
T>\frac{(2+4 L) \lambda}{c(c+\lambda)}
$$

For $L=c=1$ this yields the estimate $T>6 \lambda /(1+\lambda)$. Hence, the sampling interval has to be sufficiently large in order to allow for compensating the control effort which is reflected by the overshoot constant $C$. However, choosing a small weight in the control penalization, e.g. $\lambda=10^{-3}$, this results in a very short optimization horizon compared to the time $\bar{T}=2 L / c=2$ required for finite time controllability. 


\section{SEmilinear Reaction Diffusion Equation}

In this section we consider the reaction diffusion equation

$$
\begin{aligned}
y_{t}(x, t) & =\Delta y(x, t)-f(y(x, t)) & & \text { on } \Omega \times(0, \infty) \\
y(x, t) & =0 & & \text { on } \partial \Omega \times(0, \infty)
\end{aligned}
$$

with homogeneous Dirichlet boundary condition and initial condition $y(x, 0)=y_{0}$. The nonlinearity $f: \mathbb{R} \rightarrow \mathbb{R}$ should be continuously differentiable. Moreover, we assume $f(0)=$ 0 in order to ensure that the origin is an equilibrium. For existence and regularity results we refer to [4].

The stability behavior of this semilinear parabolic equation is characterized in the following theorem which is proven in [4].

Theorem 2: For each $\gamma \in\left(0, \lambda_{1}+f^{\prime}(0)\right)$ there exists a constant $R=R(\gamma)$ such that for all $y_{0} \in \mathcal{C}_{0}(\Omega)$ with $\left\|y_{0}\right\| \leq$ $R$ the solution $y$ of (22)-(23) satisfies

$$
\|y(t)\| \leq M\left\|y_{0}\right\| e^{-\gamma t} \quad \forall t \geq 0 .
$$

Here $\lambda_{1}=\lambda_{1}(\Omega)$ denotes the smallest eigenvalue of the differential operator $-\Delta$ in $H_{0}^{1}(\Omega)$.

Remark 4: The constant $M$ is independent of $\gamma$ and $x$. It depends only on the used norm, e. g. $M=1$ for $\|\cdot\|_{L^{2}(\Omega)}$. Furthermore the origin is unstable for $\lambda_{1}<-f^{\prime}(0)$. For details we refer to [4].

An important representative of this class is the onedimensional Chaffee-Infante equation

$$
y_{t}(x, t)=y_{x x}(x, t)+\mu\left(y(x, t)-y(x, t)^{3}\right),
$$

i.e., $f(y)=-\mu\left(y-y^{3}\right)$. For parameter $\mu=11$ and the initial condition $y(x, 0)=0.2 \sin (\pi x)$ the origin is unstable because $\lambda_{1}=\pi^{2}<11=-f^{\prime}(0)$, cf. Figure 1 .

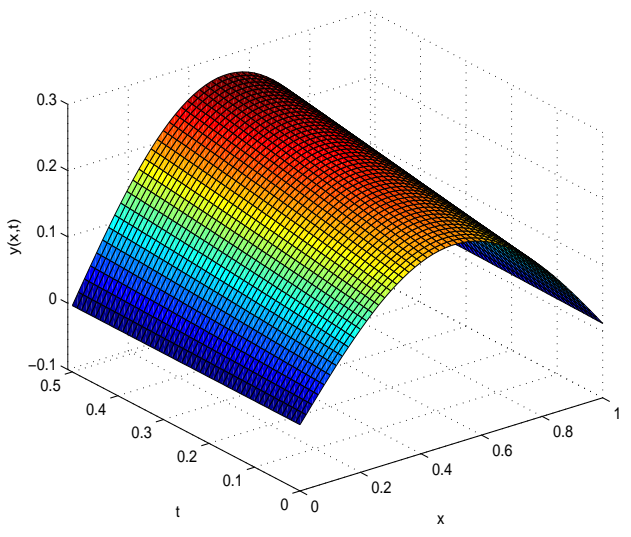

Fig. 1. Solution of the uncontrolled Chaffee-Infante equation (25) with $\mu=11$ and initial condition $y(x, 0)=0.2 \sin (\pi x)$.

Our goal consists of stabilizing Equation (22)-(23) to the origin by a receding horizon feedback. For this purpose we consider the semilinear heat equation with distributed control

$$
y_{t}(x, t)=\Delta y(x, t)-f(y(x, t))+u(x, t) .
$$

To be more precise, we do not only aim at stabilizing this semilinear parabolic PDE but give explicit estimates for minimal stabilizing horizons. To this end, we apply Theorem 2 in order to establish the (exponential) controllability condition given in Definition 1. This enables us to utilize results from Section II.

Thus, we interpret the evolution equation in consideration as a discrete time system, cf. Section III and use the standard $L^{2}$-cost functional

$$
l(y(n), u(n))=\|y(\cdot, n T)\|_{L^{2}(\Omega)}^{2}+\lambda\|u(\cdot, n T)\|_{L^{2}(\Omega)}^{2} .
$$

Existence results for this optimal control problem can be found in [9].

In order to derive Inequality (8), i.e., exponential controllability in terms of the running costs, we choose a feedback control $u(x, t):=-K y(x, t)$ with a real constant $K$. Again, we do not need the optimality of this control. We define $F(y):=f(y)+K y$ and apply the previous theorem in order to obtain

$$
l^{*}(y(n))=\|y(\cdot, n T)\|_{L^{2}(\Omega)}^{2} \leq M^{2} \sigma^{n} l^{*}(y(0))
$$

with $\sigma=e^{-2 \gamma T}$ and $\gamma=\lambda_{1}+f^{\prime}(0)+K$. Furthermore, we get

$$
\begin{aligned}
l(y(n), u(n)) & =\|y(\cdot, n T)\|_{L^{2}(\Omega)}^{2}+\lambda\|u(\cdot, n T)\|_{L^{2}(\Omega)}^{2} \\
& =\|y(\cdot, n T)\|_{L^{2}(\Omega)}^{2}+\lambda K^{2}\|y(\cdot, n T)\|_{L^{2}(\Omega)}^{2} \\
& =\left(1+\lambda K^{2}\right) l^{*}(y(n))
\end{aligned}
$$

By combining these results we obtain the desired exponential controllability

$$
l(y(n), u(n))=\left(1+\lambda K^{2}\right) l^{*}(y(n)) \leq C \sigma^{n} l^{*}(y(0))
$$

with overshoot $C:=\left(1+\lambda K^{2}\right) M^{2}$. Thus, we obtain the following theorem.

Theorem 3: Let $C:=\left(1+\lambda K^{2}\right) M^{2}$ and $\sigma=e^{-2 \gamma T}$ with $\gamma=\lambda_{1}+f^{\prime}(0)+K$. Moreover, choose $K \in \mathbb{R}^{+}$ and the optimization horizon $N \in \mathbb{N}_{\geq 2}$ such that $\alpha_{N}=$ $\alpha_{N}(C(K), \sigma(K))$ from (9) is positive then the receding horizon approach stabilizes (26),(23) with optimization horizon $N$.

Proof: Based on the estimates for $C, \sigma$ deduced in this section we apply Theorem 1 in order to obtain $\alpha \geq 0$ in (4). Moreover, in consideration of Theorem 2 choosing $\alpha_{1}(r)=$ $r^{2}$ and $\alpha_{2}(r)=c r^{2}$ with $c:=\left(1+\lambda K^{2}\right) M^{2} \sum_{k=0}^{N-1} e^{-2 \gamma k T}$ implies (6), (7). Thus, Proposition 1 shows the assertion.

Remark 5: Computing the maximal $\alpha_{N}$ for a given horizon length $N$ leads to an easy optimization problem in $K$. As a consequence, Theorem 3 allows for calculating the smallest horizon length $N$ for which Theorem 1 guarantees stability with respect to the derived parameters $C, \sigma$, cf. the result presented at the end of Section V.

Remark 6: The estimates for the minimal stabilizing horizon are in general conservative for a concrete example. The main reason for this is the estimate used in Inequality (27) which is in general conservative. Moreover, it might be possible to tighten the deduced results by choosing a more elaborated control function instead of our simple feedback control. 


\section{Numerical Results}

In this section we illustrate our theoretical results by means of numerical computations. We begin with the linear wave equation (10)-(12). The we investigate the ChaffeeInfante equation (25) as a well-known representative of the semilinear parabolic equation considered in Section IV.

\section{A. Wave equation}

We consider the wave equation (10)-(12) with the parameters $L=c=1$. The initial data are given by

$$
y_{0}(x):=\left\{\begin{array}{rll}
+2 x-0.5 & : & 0.25<x \leq 0.50 \\
-2 x+1.5 & : & 0.50<x \leq 0.75 \\
0 & : & \text { otherwise }
\end{array}\right.
$$

and $y_{1}(x) \equiv 0$. For solving the finite horizon optimal control problems we discretize the spatial domain with discretization parameter $\Delta x=0.001$ and employ the software package $\mathrm{PCC}^{2}$ in order to solve the resulting problems. Moreover, we set the sampling time $T=0.025$ and use the optimization horizon $N=2$ in this subsection.

From the relaxed Lyapunov inequality (4) it follows that we need a decay of the running cost in each time step for guaranteeing instantaneous control. Figure 2 depicts the finite horizon optimal value function $V_{2}(\cdot)$ for $\omega_{1}=\omega_{2} \equiv 1$, i.e., the classical energy, and for the weighted energy defined in (14). Apparently each of these two curves is monotonically decreasing, but only the trajectory corresponding to (15) seems to be strictly monotonically decreasing.

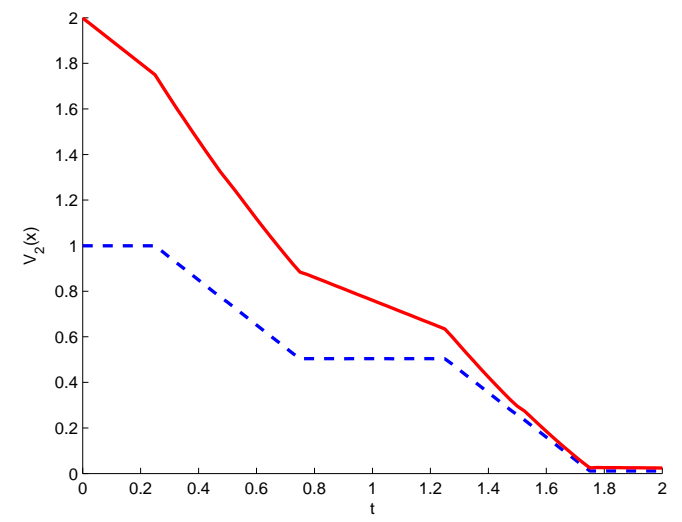

Fig. 2. Comparison of the optimal value function $V_{2}(\cdot)$ for different weight functions in the running costs $l(\cdot, \cdot)$. The dashed curve corresponds to the classical energy norm, i.e., $\omega_{1}=\omega_{2} \equiv 1$. Whereas the continuous curve is based on our weighted energy from (15).

In order to investigate this more accurately we calculate the corresponding $\sigma$-values. In order to visualize our theoretically calculated estimate we have drawn a horizontal line at $1-T / 3$ in Figure 3 which shows that the calculated values for the classical energy are arbitrarily close to one and exceed our estimated bound whereas the values corresponding to (15) are smaller than $1-T / 3$ which confirms our theoretical results.

\footnotetext{
${ }^{2}$ see http://www.nonlinearmpc.com/
}

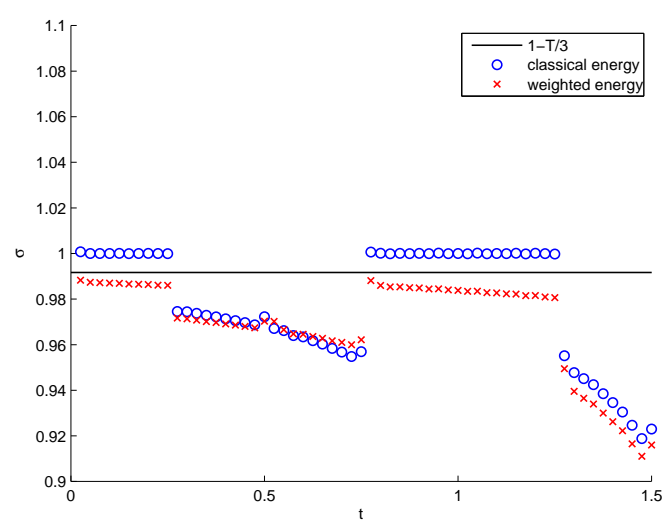

Fig. 3. We depict the corresponding $\sigma$-values with respect to the classical (o) and the weighted energy $(15, x)$ from Inequality (19). Moreover, we have drawn a horizontal line at $1-T / 3$ in order to indicate our theoretically derived bound.

The solution trajectory of the instantaneous controlled wave equation is depicted in Figure 4. Hence, receding horizon control with $N=2$ performs very well for our stabilization task. The computing time for solving the instantaneous control problem on the time interval $[0,2]$ is less than one second even for a fine spatial discretization.

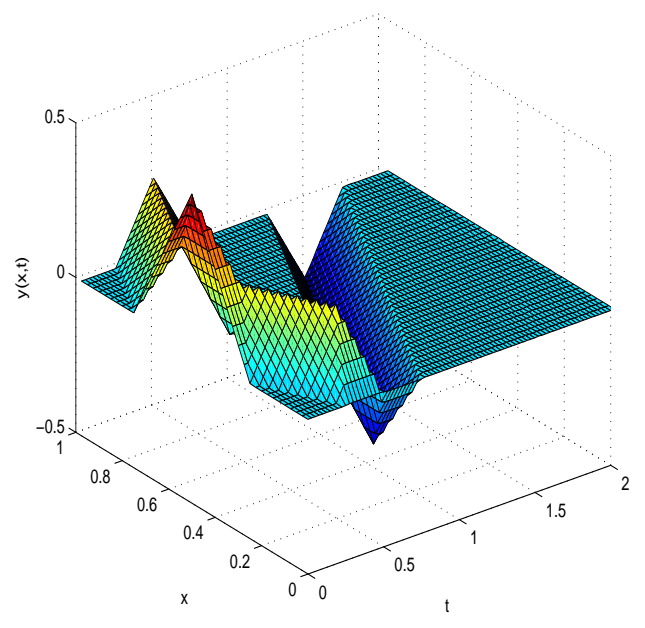

Fig. 4. The solution trajectory for the instantaneous controlled wave equation (optimization horizon $N=2$ ) for the initial data given in Subsection V-A.

\section{B. Chaffee-Infante equation}

In this subsection we investigate a semilinear parabolic evolution equation, the Chaffee-Infante equation (25), with parameter $\mu=11$, initial data

$$
y(x, 0)=0.2 \sin (\pi x)
$$

and Dirichlet boundary conditions, i.e., $y(0, t)=y(1, t)=0$. Since $\lambda_{1}=\pi^{2}<11=-f^{\prime}(0)$ the origin is unstable for the uncontrolled equation, cf. Section IV and Figure 1. 
We choose the discretization parameter $T=0.01$ and the regularization parameter $\lambda=0.01$. Furthermore, we use the spatial discretization $\Delta x=0.01$ for our numerical results. Figure 5 indicates that the receding horizon approach does not stabilize the system at $y^{*} \equiv 0$ for $N=3$. Indeed, it resembles the shape of the uncontrolled equation, cf. Figure 1.

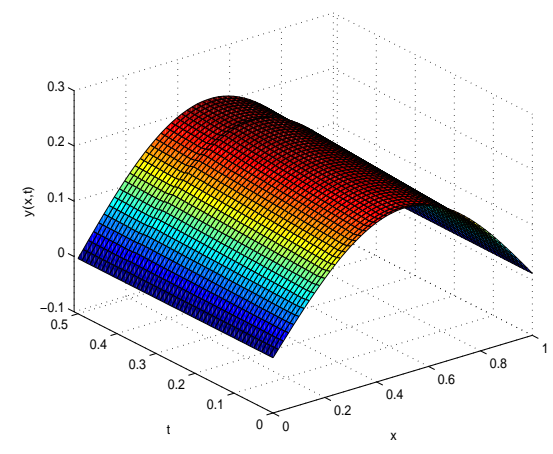

Fig. 5. Solution of the Chaffee-Infante equation (25) with distributed receding horizon control with optimization horizon $N=3$.

Enlarging the horizon to $N=6$ relocates the obtained profil in the direction of the desired equilibrium. However, the algorithm still is not able to stabilize the system, cf. Figure 6. In [2] it has been shown numerically how to design appropriate running costs $l$ such that the stabilizing horizon is shortened without the explicit knowledge of $C$ and $\sigma$. In contrast to that, we have deduced estimates for these parameters in Section IV which enable us to employ Theorem 3 in order to compute a concrete minimal stabilizing horizon, i.e., the smallest $N \in \mathbb{N}_{\geq 2}$ with $\alpha_{N}>0$.

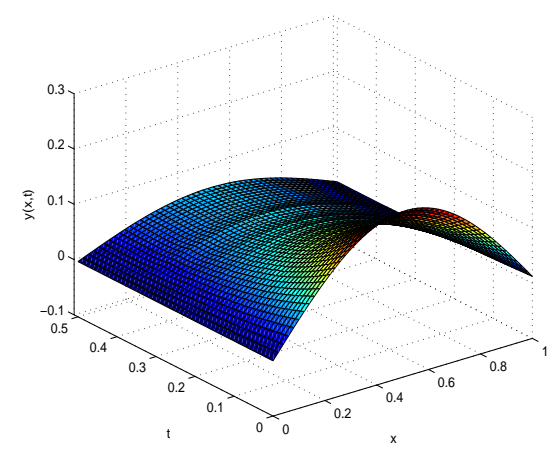

Fig. 6. Solution of the Chaffee-Infante equation (25) with distributed receding horizon control with optimization horizon $N=6$.

Proceeding as described in Remark 5 we obtain $N=10$ as the minimal stabilizing horizon. The computed (optimal) choice for the constant $K$ is 2.45 . Consequently, we obtain $\alpha=0.014>0$ from Theorem 3 with parameters $C=1.06$ and $\sigma=0.97$. Figure 7 confirms our theoretically obtained results.

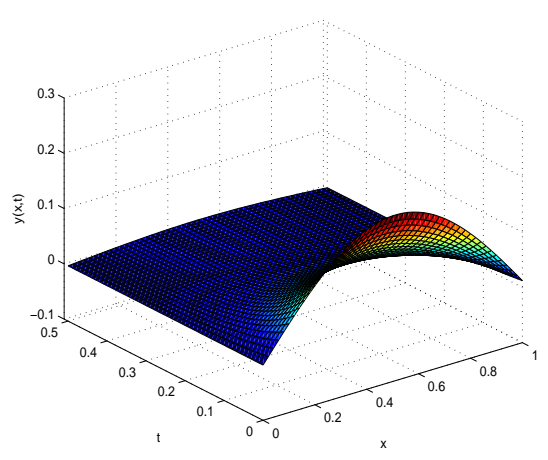

Fig. 7. Solution of the Chaffee-Infante equation (25) with distributed receding horizon control with optimization horizon $N=10$, i.e., the minimal stabilizing horizon.

\section{CONClusion}

In this paper we have shown that the one dimensional linear wave equation is intantaneous controllable, i.e., stabilizable by receding horizon optimal control with horizon $N=2$ for appropriate running costs. For future work it will be interesting to generalize these results to multidimensional and semilinear wave equations.

Furthermore, we presented stability and suboptimality results for a semilinear parabolic equation. Since these estimates are in general conservative, in future work we will implement an adaptive receding horizon controller similar to the ODE approach in [10].

\section{REFERENCES}

[1] N. Altmüller, L. Grüne, and K. Worthmann. Instantaneous control of the linear wave equation. Proceedings of MTNS 2010, Budapest, Hungary, to appear, 2010.

[2] N. Altmüller, L. Grüne, and K. Worthmann. Performance of nmpc schemes without stabilizing terminal constraints. to appear, 2010.

[3] T.A. Badgwell and S.J. Qin. A survey of industrial model predictive control technology. Control Engineering Practice, 11:733-764, 2003.

[4] T. Cazenave and A. Haraux. An Introduction to Semilinear Evolution Equation. Oxford Science Publications, 1998.

[5] L. Grüne. Analysis and design of unconstrained nonlinear mpc schemes for finite and infinite dimensional systems. SIAM J. Control Optim., 48:1206-1228, 2009.

[6] L. Grüne, J. Pannek, M. Seehafer, and K. Worthmann. Analysis of unconstrained nonlinear mpc schemes with varying control horizon. Preprint, Universität Bayreuth, submitted, 2009.

[7] R. Hundhammer and G. Leugering. Online Optimization of Large Scale Systems, chapter Instantaneous Control of Vibrating String Networks, pages 229-249. Springer, 2001.

[8] K. Ito and K. Kunisch. Receding horizon optimal control for infinite dimensional systems. ESAIM: COCV, 8:741-760, 2002.

[9] P. Naittaanmäki and D. Tiba. Optimal Control Of Nonlinear Parabolic Systems. Marcel Dekker, Inc, 1994.

[10] J. Pannek. Receding horizon control: A suboptimality-based approach. PhD Thesis, University of Bayreuth, 2009.

[11] J. Rawlings and D. Mayne. Model Predictive Control: Theory and Design. Nob Hill Publishing, 2009.

[12] W. Strauss. Partial Differential Equations: An Introduction. Wiley, 1992.

[13] E. Zuazua. Optimal and approximate control of finite-difference approximation schemes for the 1-d wave equation. Rendiconti di Matematica, Serie VIII, pages 201-237, 2004. 